\title{
DESIGN AND DEVELOPMENT OF AN ELECTRON RECIRCULATOR FOR STUDY OF HIGH-CURRENT BEAM DYNAMICS ${ }^{\dagger}$
}

\author{
J.G. Wang, S. Bernal, P. Chin, J.J. Deng a , Y. Li, M. Reiser, H. Suk ${ }^{\text {}}$, M. Venturini, Y. Zou, \\ Institute for Plasma Research, University of Maryland, College Park, MD 20742 \\ T. Godlove, FM Technologies, Inc. \\ R. C. York, NSCL, Michigan State University
}

\begin{abstract}
An electron recirculator is being developed at the University of Maryland for studying the physics of space-charge dominated beams in circular systems. The E-ring has been designed to transport an electron beam of $10 \mathrm{keV}, 100 \mathrm{~mA}, 50 \mathrm{~ns}$ in a smooth circular lattice of $11.5 \mathrm{~m}$ in circumference, focused by 72 printed-circuit (PC) quadrupole FODO elecments, and deflected by 36 PC dipoles. This paper reports the progress and status of the E-ring project.
\end{abstract}

\section{INTRODUCTION}

Rapid-cycling rings and other recirculator systems operating with intense beams beyond the conventional space-charge limit are of great interest for applications in high-energy physics accelerators such as high-intensity booster synchrotrons and muon colliders, heavy-ion inertial fusion drivers, spallation neutron sources, and other fields. There is very little theoretical or experimental knowledge that would allow us to make any predictions on the beam behavior in such novel systems.

The history of high currents in conventional synchrotrons has been dominated by the necessity for a very large number of revolutions of the beam and the danger of crossing ring resonances slowly. Previous studies $[1,2]$ have shown that it may be possible to exceed the limiting beam current - the Laslett tune shift limit - by a large factor, provided the number of turns is limited to perhaps 100 . The natural solution is then a rapid cycling ring or recirculator.

We have been developing a small electron ring at the University of Maryland for studying the evolution of a space-charge dominated beam in a circular lattice [3]. The important physics issues to be addressed by the Ering are limits on the number of turns, resonance traversal, maximum transport current, thermal equilibrium and equipartitioning, bending of spacecharge dominated beams and dispersion [4] in circular lattices, space-charge waves and longitudinal instabilities, etc. The E-ring will also provide a longer path for the experimental study of the physics of spacecharge dominated beams than is available in our existing 5-m long transport channel.

' Research supported by the US Department of Energy.

\section{DESIGN CONSIDERATIONS}

The schematic of the E-ring layout is shown in Fig. 1. A $10 \mathrm{keV}, 100 \mathrm{~mA}, 30-75 \mathrm{~ns}$ electron beam with a normalized effective emittance of $10 \mathrm{~mm}$-mrad from our existing electron gun is injected into the ring with the aid of a pulsed, Panofsky-type quadrupole, and a fast deflecting dipole. The ring design is based on a "woundup" linear channel. Transverse focusing is provided by 70 printed-circuit quadrupoles (plus two Panofsky quads of special design). Three induction gaps are employed for longitudinal focusing of both parabolic and rectangular bunches. The diagnostics along the E-ring include three resistive beam position monitors, three capacitive BPMs, three retarding field beam energy analyzers, and three phosphor screen beam image viewers. A pulsed extraction system similar to the injector is included for beam analysis in the end chamber housing an emittance meter and an energy analyzer.

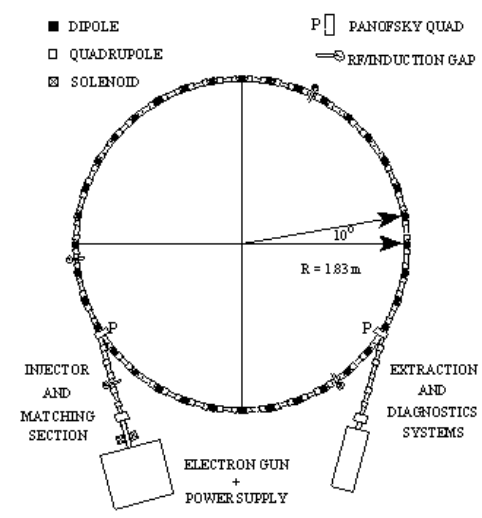

Fig. 1 Layout of the E-ring design.

A distinct feature in the transport of space-charge dominated beams is that the focusing elements have to be packed very densely along the channel. In the E-ring design, the PC quadrupole length is $4.4 \mathrm{~cm}$, while the half-lattice length is $16 \mathrm{~cm}$. For a focusing strength which corresponds to the phase advance $\sigma_{0}=76^{\circ}$ without space-charge, the mean beam radius is $1.04 \mathrm{~cm}$ and the ripple factor is 0.7 , according to the smooth approximation theory [5]. The bore diameter of the 
transport channel is chosen to be $4.90 \mathrm{~cm}$. The spacecharge depresses the tune by a factor of more than 5 . The lap time is $197 \mathrm{~ns}$ which is sufficient to allow for pulsing of injector elements, and extraction into a diagnostic chamber after any given number of turns.

The ring would initially operate in a transport mode, with single-bunch injection, minimum dispersion. The future acceleration of beams up to $50 \mathrm{keV}$ through the induction gaps is also considered in the design. The wall thickness is chosen to be $0.5 \mathrm{~mm}$ and the dipole mandrels are designed with non-metallic material, so that the magnetic field can penetrate fast enough into the pipe during acceleration.

The ring main parameters are summarized in Table I.

TABLE I Design Parameters for the E-Ring

$\begin{array}{ll}\text { Injection Energy } & 10 \mathrm{keV} \\ \text { Injection Current } & 100 \mathrm{~mA} \\ \text { Generalized Perveance } & 0.0015 \\ \text { Initial Emittance } & 10 \mathrm{~mm}-\mathrm{mrad} \\ \text { Mean Beam Radius } & 1.04 \mathrm{~cm} \\ \text { Vacuum Tube Bore Diameter } & 4.90 \mathrm{~cm} \\ \text { Circumference } & 11.5 \mathrm{~m} \\ \text { Lap Time } & 197 \mathrm{~ns} \\ \text { Lattice Periods } & 36 \\ \text { Half-Lattice Length } & 16 \mathrm{~cm} \\ \text { Quadrupole Length } & 4.4 \mathrm{~cm} \\ \text { Quadrupole Diameter } & 5.3 \mathrm{~cm} \\ \text { Gradient } & 8.1 \mathrm{G} / \mathrm{cm} \\ \text { Tune } v_{0} & 7.6 \\ \text { Phase advance w/o space charge } \sigma_{0} & 76^{\circ} \\ \text { Phase advance w/ space charge } \sigma & 9^{\circ}-25^{\circ}\end{array}$

\section{MAGNET DESIGN}

Due to the low energy of electron beams in the Ering the magnetic field strength required to focus the beams in a FODO lattice and to bend the beams in a small angle is very moderate. We have decided to employ short magnetic quadrupoles and dipoles made of current loops on flexible printed circuits. This approach not only can meet satisfactory performance for beam dynamics in the E-ring, but also can achieve substantial economic savings.

The design of a model PC quadrupole was reported in the 1995 PAC conference [6]. Some revisions have been made ever since. The new PC quadrupoles have an aspect ratio of 0.83 as indicated in Table I. The calculated radial linearity of the axially-integrated field is satisfactory. For radii through $0.7 \mathrm{R}$, where $\mathrm{R}$ is the quadrupole radius, the largest deviation is $0.13 \%$. The required current to produce the focusing field is $1.7 \mathrm{~A}$.

The PC dipole design is based on the same method as for the PC quadrupoles, except that the number of current loops is only half of that for the quadrupoles. The calculated maximum deviation from linearity is found to be $0.35 \%$ at $0.7 \mathrm{R}$. The aspect ratio has been chosen to be unity.

\section{SINGLE-PARTICLE DYNAMICS}

The lattice of the E-ring employs short magnetic elements. Since this introduces nonlinearities in the transverse dynamics of the charged particles, transfer maps have been constructed to take this effect into account. These maps have been used to simulate singleparticle motion and determine the dynamic aperture of the E-ring by using MaryLie 3.0, a particle code based on Lie algebraic methods.

The single-particle study has investigated the effects of those nonlinearities in terms of dynamic aperture. For the stability studies, the linear fields of the E-ring quadrupoles and dipoles are used and then compared to the results when non-linear fringe fields are included. The simulation has showed the satisfactory dynamic aperture of the E-ring design. The stability domain in the transverse plane (controlled by the nonlinearities arising from extended fringe fields) is large enough to include the physical aperture of the vacuum pipe [7].

We have also extended the simulation studies of single-particle dynamics to include the effects of random errors in the magnetic fields due to magnet mispowering or misplacement and an imperfectly compensated earth's magnetic field. The results in the case of mispowered magnets show only a modest erosion of the dynamic aperture. This study has provided acceptable tolerances on the mechanical design.

\section{INJECTION AND EXTRACTION}

The E-ring is designed to have a single bunch injection and to operate in a transport mode. The injector consists of a gridded electron gun, a straight matching section, a Panofsky quadrupole, and a fast deflecting dipole. The straight matching section contains a short solenoidal lens and two periods of the PC FODO quadrupoles. The Panofsky quadrupole, chosen to handle the special geometric problem in that region, is a regular focusing element on the ring. Its magnetic field should be off during injection, and well established before the beam comes back after one turn, i.e., after 197 ns. Thus, this quadrupole should be a special design, not only for its elongated shape but also for its fast rising time. The fast deflecting dipole performs two functions: it deflects beams by $10^{\circ}$ counter-clockwise during injection, and afterwards, it should change its field polarity within the $197 \mathrm{~ns}$ lap time and deflect the beam $10^{\circ}$ clockwise during the beam life time. This dipole should also have very fast rise time.

The design of the injection/extraction, as well as the fast defecting dipole and the Panofsky quadrupole is detailed in an accompanying paper in these Proceedings [8]. We also have an experimental program in progress to study beam transport in two periods of a PC 
quadrupole lattice, and bending [9]. This experiment will include bending of beams, and will provide necessary information to validate the injection design.

\section{LONGITUDINAL FOCUSING}

The longitudinal bunch profiles envisioned in the electron recirculator include parabolic bunches and rectangular bunches. For focusing parabolic bunches, an external linear field along the bunch is required. On the other hand, focusing rectangular bunches requires socalled "ear" field. We have designed longitudinal focusing elements for controlling of bunch profiles in the electron ring. Three induction modules with different gap-voltage waveforms will be placed $120^{\circ}$ apart along the ring to fulfill this task.

The longitudinal dynamics of the E-ring, the requirement for focusing both parabolic and rectangular bunches, the design and development of the induction module and its modulators are described in another paper in these Proceedings [10].

\section{DIAGNOSTICS}

The diagnostics along the electron ring include three resistive beam position/current monitors, three capacitive beam pick-ups, three retarding field energy analyzers, and three phosphor screens for beam viewing. In addition, a diagnostic chamber at the end of the extraction line will also be installed. The BPMs should provide a sensitivity of better than $10 \mathrm{mV}$ for the beam current and a rise time of less than $1 \mathrm{~ns}$. The development of the resistive BPMs is described in another paper in these Proceedings [11].

In addition, three capacitive beam position monitors (BPM) will also be employed. Capacitive pick-ups do not require magnetic ferrite for insulation, thus, they can be put in vacuum with little out-gassing. Capacitive probes have the advantage of higher sensitivity, hence better signal-to-noise ratio. They may in fact be the only way to diagnose small beam currents, e.g., beams required for initial tune-up free from space-charge effects. On the other hand they have poorer frequency response. Thus we propose that it is wise to have both resistive and capacitive BPMs available.

The design of the energy analyzers and phosphor image viewers is straight-forward following the design of our existing devices.

\section{MECHANICAL DESIGN}

Mechanically, the ring consists of 18 sections plus the injection and extraction. Each section contains two $10^{\circ}$ bends and two periods of FODO elements. Nine sections contain vacuum/diagnostic chambers each, to obtain a vacuum in the range of $10^{-10}$ torr. The vacuum/diagnostic chambers also house the diagnostics such as three capacitive pick-ups, three energy analyzers, and three phosphor screen image viewers. A major advantage of the vacuum/diagnostic chambers is their flexibility. The BPMs and energy analyzers can be modified and reinstalled without major disruption to the E-ring. Other nine sections contain three induction gaps, three resistive BPMs, and two locations for the injection and extraction. The main consideration for two different kinds of sections is that the induction gaps and resistive BPMs require magnetic cores and other components, so that it would be difficult to install them inside the vacuum/diagnostic chambers.

\section{EARTH MAGNETIC FIELD}

An important problem in our ring design is the effect of the earth's magnetic field due to the relatively low beam energy and hence the low focusing and bending field. Under the action of the vertical component of the earth's field alone, a $10 \mathrm{keV}$ electron is deflected by about $2 \mathrm{~mm}$ in a half-lattice distance. This problem has been considered seriously since the start of this project. The remedy includes cancellation by external current-carrying wires, compensation by steering magnets, and/or shielding by mu-metals. We intend to orient the direction of orbital motion so that the earth's field assists in the bending. The dipoles themselves will also be used for further orbit control.

\section{REFERENCES}

[1] M. Month and W.T. Weng, 12th Int-1 Accel. Conf., 324 (1983); see also review article by W.T. Weng, AIP Conf. Proc. 153, 349 (1987).

[2] I. Hofmann and K. Beckert, IEEE Trans. Nucl. Sci. NS-32, 2264 (1985).

[3] M. Reiser, S. Bernal, A. Dragt, M. Venturini, J. G. Wang, H. Onishi, and T. F. Godlove, Fusion Engineering and Design 32-33, 293 (1996).

[4] M. Venturini and M. Reiser, in these Proceedings.

[5] M. Reiser, Theory and Design of Charged Particle Beams (John Wiley, New York, 1994).

[6] T.F. Godlove, S. Bernal, M. Reiser, in the Proc. of Part. Accel. Conf., 2117 (1995).

[7] M. Venturini, S. Bernal, A. Dragt, M. Reiser, J.G. Wang and T.F. Godlove, Fusion Engineering and Design 32-33, 283 (1996).

[8] T. Godlove, S. Bernal, J. J. Deng, Y. Li, M. Reiser, J. G. Wang, and Y. Zou, in these Proceedings.

[9] S. Bernal, A. Dragt, M. Reiser, M. Venturini, J. G. Wang, and T. F. Godlove, Fusion Engineering and Design 32-33, 277 (1996).

[10] J. J. Deng, J. G. Wang, M. Reiser, and T. Godlove, in these Proceedings.

[11] H. Suk, J. G. Wang, S. Bernal, and M. Reiser, in these Proceedings.

a. Permanent Address: PO.Box 523-56, Cheng-Du, China. b. Present Address: Dept. of Physics, UCLA. 